\title{
NONEXISTENCE OF CONTINUOUS SELECTIONS OF THE METRIC PROJECTION FOR A CLASS OF WEAK CHEBYSHEV SPACES
}

BY

MANFRED SOMMER

\begin{abstract}
The class $\mathfrak{B}$ of all those $n$-dimensional weak Chebyshev subspaces of $C[a, b]$ whose elements have no zero intervals is considered. It is shown that there does not exist any continuous selection of the metric projection for $G$ if there is a nonzero $g$ in $G$ having at least $n+1$ distinct zeros. Together with a recent result of Nürnberger-Sommer, the following characterization of continuous selections for $\mathfrak{B}$ is valid: There exists a continuous selection of the metric projection for $G$ in $\mathfrak{B}$ if and only if each nonzero $g$ in $G$ has at most $n$ distinct zeros.
\end{abstract}

If $G$ is a nonempty subset of a normed linear space $E$, then for each $f$ in $E$ we define $P(f):=\left\{g_{0} \in G \mid\left\|f-g_{0}\right\|=\inf \{\|f-g\| \mid g \in G\}\right\}$. P defines a set-valued mapping of $E$ into $2^{G}$ which in the literature is called the metric projection onto $G$. A continuous mapping $s$ of $E$ onto $G$ is called a continuous selection for the metric projection $P$ (or, more briefly, continuous selection) if $s(f)$ is in $P(f)$ for each $f$ in $E$. In this paper we treat the problem of the existence of continuous selections for $n$-dimensional subspaces $G$ of $C[a, b]$, with $C[a, b]$, as usual, the Banach space of real-valued continuous functions on $[a, b]$ under the uniform norm.

A. Lazar, P. Morris and D. Wulbert [3] have characterized the 1-dimensional subspaces of $C(X)$ with $X$ compact Hausdorff, which admit a continuous selection. They have raised the problem of characterizing the corresponding $n$-dimensional subspaces.

Using the kind of selection established by Lazar-Morris-Wulbert, it does not seem possible to get a general theorem for $n$-dimensional subspaces of $C[a, b]$. With new methods, however, and in the setting of weak Chebyshev subspaces, Nürnberger-Sommer [4], [5] and Sommer [7], [8] have given both sufficient conditions for the existence of continuous selections and characterization theorems of the existence of continuous selections for several classes of $n$-dimensional weak Chebyshev subspaces of $C[a, b]$ (see also Nürnberger [6]).

In the following we refer to a result in [4].

Nürnberger-Sommer have shown that for those weak Chebyshev spaces $G$ whose elements $g(g \neq 0)$ have at most $n$ distinct zeros on $[a, b]$, there exists exactly one continuous selection.

Here we show that for those weak Chebyshev spaces $G$ which have no elements

Received by the editors March 22, 1978.

AMS (MOS) subject classifications (1970). Primary 41A30, 46E15.

Key words and phrases. Continuous selection, metric projection, weak Chebyshev spaces. 
vanishing on intervals but which have elements $g(g \neq 0)$ with at least $n+1$ distinct zeros, there does not exist any continuous selection. To prove this result we apply a fundamental lemma of Lazar-Morris-Wulbert.

Hence we have the following characterization of the existence of continuous selections for those $n$-dimensional weak Chebyshev subspaces $G$ of $C[a, b]$ whose elements do not vanish on intervals.

There exists a continuous selection for $G$ if and only if each $g$ in $G$ has at most $n$ distinct zeros.

In the following let $G$ be an $n$-dimensional subspace of $C[a, b]$.

1. Definition. $G$ is called weak Chebyshev if each $g$ in $G$ has at most $n-1$ changes of sign, i.e. there do not exist points $a \leqslant x_{0}<x_{1}<\cdots<x_{n} \leqslant b$ such that $g\left(x_{i}\right) \cdot g\left(x_{i+1}\right)<0, i=0, \ldots, n-1$.

R. C. Jones and L. A. Karlovitz have characterized these spaces. For this characterization we need the following definition.

2. Definition. If $f$ is in $C[a, b]$, then $g$ in $P(f)$ is called an alternation element (AE) of $f$ if there exist $n+1$ distinct points $a \leqslant x_{0}<x_{1}<\cdots<x_{n}<b$ such that $\varepsilon(-1)^{i}(f-g)\left(x_{i}\right)=\|f-g\|, i=0, \ldots, n, \varepsilon= \pm 1$. The points $x_{0}, \ldots, x_{n}$ are called alternating extreme points of $f-g$.

Jones and Karlovitz [1] have proved the following theorems.

3. TheOREM. $G$ is weak Chebyshev if and only if for each $f$ in $C[a, b]$ there exists at least one $A E$ in $P(f)$.

4. THEOREM. $G$ is weak Chebyshev if and only if given $a=x_{0}<x_{1}<\cdots<x_{n-1}$ $<x_{n}=b$ there exists a $g$ in $G, g \neq 0$, such that

$$
(-1)^{i+1} g(x) \geqslant 0, \quad x_{i-1}<x<x_{i}, i=1, \ldots, n .
$$

In order to show the nonexistence of a continuous selection for a class of weak Chebyshev spaces, we need the following standard definition.

5. Definition. A zero $x_{0}$ of $f$ in $C[a, b]$ is said to be a simple zero if $f$ changes sign at $x_{0}$ or if $x_{0}=a$ or $x_{0}=b$. A zero $x_{0}$ of $f$ in $C[a, b]$ is said to be a double zero if $f$ does not change sign at $x_{0}$ and $x_{0}$ is in $(a, b)$. Let $Z(f):=\{x \in[a, b] \mid f(x)=$ 0 ) the set of zeros of $f$.

We denote by $|Z(f)|$ the number of distinct zeros of $f$ and by $\left|Z^{*}(f)\right|$ the number of zeros of $f$, counting simple zeros as one zero and double zeros as two zeros.

6. Definition. Let $x_{1}, x_{2}$ be zeros of $f$ in $C[a, b]$. These zeros are said to be separated if there is a $y_{1}$ in $[a, b]$ with

$$
x_{1}<y_{1}<x_{2}, \quad f\left(y_{1}\right) \neq 0 .
$$

A zero $x_{0}$ of $f$ in $G$ is said to be an essential zero with respect to $G$, if there is a $g$ in $G$ with $g\left(x_{0}\right) \neq 0$.

Nürnberger-Sommer [4] have given the following sufficient condition for the existence of a continuous selection. 
7. Theorem. Let $G$ be weak Chebyshev. Let $|Z(g)|<n$ for each $g$ in $G, g \neq 0$. Then there exists exactly one continuous selection.

Since $|Z(g)| \leqslant n$ for each $g$ in $G, g \neq 0$, no $g$ in $G$ has a zero interval. We will show that for those weak Chebyshev spaces $G$ which have no elements with zero intervals but which have elements $g$ with $|Z(g)| \geqslant n+1$, there does not exist any continuous selection. For this we need the following lemmas.

8. LemMa (LAZAR-MorRIS-WULBERT [3]). If $s$ is a continuous selection of $C[a, b]$ onto $G$ and $f$ is in $C[a, b],\|f\|=1$ and 0 is in $P(f)$, then there is a $g_{0}$ in $P(f)$ such that

(1) for every $x \in$ bd $Z(P(f)) \cap f^{-1}(1)$ and every $g$ in $P(f)$ there is a neighborhood $U$ of $x$ for which $g_{0} \geqslant g$ on $U$ and

(2) for every $x \in$ bd $Z(P(f)) \cap f^{-1}(-1)$ and every $g$ in $P(f)$ there is a neighborhood $V$ of $x$ for which $g_{0} \leqslant g$ on $V$.

Here let $Z(P(f)):=\{x \in[a, b] \mid g(x)=0$ for each $g$ in $P(f)\}$ and bd $Z(P(f))$ is the set of boundary points of $Z(P(f))$ under the topology of $[a, b]$.

9. Lemma (Stockenberg [9]). Let $G$ be weak Chebyshev. Then no $g$ in $G$ has more than $n$ separated, essential zeros and if there is $a g$ in $G$ with $n$ separated, essential zeros $x_{1}<\cdots<x_{n}$, then $g(x)=0$ for all $x$ in $\left[a, x_{1}\right] \cup\left[x_{n}, b\right]$.

Now we are able to prove the nonexistence of a continuous selection for a class of weak Chebyshev spaces.

10. Theorem. Let $G$ be weak Chebyshev. Let no $g$ in $G(g \neq 0)$ have a zero interval but let a nontrivial function $\tilde{g}_{0}$ be in $G$ such that $\left|Z\left(\tilde{g}_{0}\right)\right| \geqslant n+1$. Then there does not exist any continuous selection.

Proof. Let $a \leqslant z_{0}<z_{1}<\cdots<z_{n} \leqslant b$ be $n+1$ distinct zeros of $\tilde{g}_{0}$.

First case: $a \leqslant z_{0}, z_{n}<b$. We will construct a function $g_{0}$ having exactly $n-1$ zeros with changes of sign and two further zeros on $[a, b)$. Since $z_{0}, \ldots, z_{n}$ are separated zeros of $\tilde{g}_{0}$, by Lemma 9 , there are two points $z_{i}, z_{j} \in\left\{z_{0}, \ldots, z_{n}\right\}, i<j$, such that

$$
g\left(z_{i}\right)=g\left(z_{j}\right)=0 \text { for all } g \in G .
$$

We choose $n-1$ distinct points

$$
z_{n}<t_{1}<\cdots<t_{n-1}<b .
$$

By Theorem 4 there exists a $g_{0} \in G, g_{0} \neq 0$, such that

$$
(-1)^{i+1} g_{0}(x) \geqslant 0, \quad t_{i-1}<x<t_{i}, i=1, \ldots, n, t_{0}=a, t_{n}=b .
$$

Moreover $g_{0}\left(z_{i}\right)=g_{0}\left(z_{j}\right)=0$.

Since $t_{1}, \ldots, t_{n-1}$ are zeros with changes of sign of $g_{0}$ and $G$ is weak Chebyshev of dimension $n$, the function $g_{0}$ has no further change of sign on $(a, b)$ and, therefore, no change of sign at $z_{i}$ and $z_{j}$.

Let $\left\|g_{0}\right\| \leqslant 1$. We choose $n+1$ distinct points $\left\{v_{l}\right\}_{l=0}^{n}$ satisfying

$$
z_{i}<v_{0}<z_{j}<v_{1}<t_{1}<v_{2}<\cdots<t_{n-1}<v_{n}<b \text {. }
$$


We choose $\varepsilon>0$ such that

$$
\left\{z_{i}, z_{j}, t_{1}, \ldots, t_{n-1}, b\right\} \cap\left[v_{l}-\varepsilon, v_{l}+\varepsilon\right]=\varnothing, \quad l=0, \ldots, n .
$$

Now we construct an $f \in C[a, b]$ as follows:

(a)

$$
\begin{aligned}
& f\left(z_{i}\right)=1, \quad f\left(z_{j}\right)=-1 \\
& f(x)=1 \text { for all } x \in\left[v_{0}-\varepsilon, v_{0}+\varepsilon\right] \\
& f(x)=(-1)^{l+1} \text { for all } x \in\left[v_{l}-\varepsilon, v_{l}+\varepsilon\right], l=1, \ldots, n
\end{aligned}
$$

(b)

$$
\max \left\{-1+g_{0}(x),-1\right\} \leqslant f(x) \leqslant \min \left\{1+g_{0}(x), 1\right\} \quad \text { for all } x \in[a, b] .
$$

Then $\|f-0\|=\left\|f-g_{0}\right\|=1$. Because of $g\left(z_{i}\right)=0$ for all $g \in G$, we always get $f\left(z_{i}\right)-g\left(z_{i}\right)=1$ and, therefore, 0 and $g_{0}$ are elements of $P(f)$.

Now let $g \in P(f), g \neq 0$. For each $l \in\{1, \ldots, n\}$ there exists a $y_{l} \in\left[v_{l}-\varepsilon, v_{l}\right.$ $+\varepsilon]$ such that $(-1)^{l+1} g\left(y_{l}\right)>0$. Hence the function $g$ has at least $n-1$ changes of sign on $\left(v_{1}-\varepsilon, b\right)$. Then $g \geqslant 0$ on $\left[a, v_{1}-\varepsilon\right]$.

Therefore the function $g$ has a double zero in $z_{j}$ and also in $z_{i}$, if $z_{i}>a$.

Since no $g \in G$ has a zero interval, it follows that $z_{i}, z_{j} \in \operatorname{bd} Z(P(f))$. Now we apply Lemma 8.

If there exists a continuous selection, then there exists a $\tilde{g} \in P(f)$ such that

(i) for $z_{i}$ and $g_{0}$ there is a neighborhood $U$ of $z_{i}$ for which $\tilde{g}>g_{0}$ on $U$, and

(ii) for $z_{j}$ and 0 there is a neighborhood $V$ of $z_{j}$ for which $\tilde{g}<0$ on $V$.

Since $\tilde{g} \geqslant g_{0}$ on $U, \tilde{g} \neq 0$.

Moreover, $\tilde{g} \geqslant 0$ on $\left[a, v_{1}-\varepsilon\right]$. Therefore, in every neighborhood $V$ of $z_{j}$ there is a point $\tilde{x}$ such that $\tilde{g}(\tilde{x})>0$. But this is a contradiction to Lemma 8 .

Second case: $a<z_{0}, z_{n} \leqslant b$. We can treat this case analogously.

Third case: Let $|Z(g)| \leqslant n$ on $[a, b)$ and on $(a, b]$ for all $g \in G$. By hypothesis there is a $\tilde{g}_{0} \in G, \tilde{g}_{0} \neq 0$, with exactly $n+1$ distinct zeros $a=z_{0}<z_{1}<\cdots<$ $z_{n}=b$. Let $a<t_{1}<t_{2}<\cdots<t_{k}<b$ be all zeros with changes of sign and $a<y_{1}<y_{2}<\cdots<y_{n-k-1}<b$ be all double zeros of $\tilde{g}_{0}$.

First we show that $k=n-1$ or $k=n-2$. No other possibilities are allowed.

We assume that $k \leqslant n-3$. We choose $n-k-1$ points $z_{n-1}<t_{k+1}<\cdots<$ $t_{n-1}<b$. By Theorem 4 there exists a $g_{0} \in G, g_{0} \neq 0$, such that

$$
(-1)^{i+1} g_{0}(x) \geqslant 0, \quad t_{i-1}<x<t_{i}, i=1, \ldots, n, t_{0}=a, t_{n}=b .
$$

We may assume that $g_{0} \cdot \tilde{g}_{0} \geqslant 0$ on $\left[a, t_{k+1}\right]$. Since $n-k>3, \tilde{g}_{0}$ has at least $n-k-1 \geqslant 2$ double zeros on $(a, b)$ and therefore $\left|Z^{*}\left(\tilde{g}_{0}\right)\right|>n+1$ on $(a, b)$. If $g_{0}\left(y_{i}\right) \neq 0$ for all $i \in\{1, \ldots, n-k-1\}$, then for sufficiently small $c>0$ the function $\tilde{g}_{0}-c g_{0}$ has at least $n+1$ changes of sign. This is a contradiction of the hypothesis on $G$.

If there are $i_{1}, i_{2} \in\{1, \ldots, n-k-1\}$ such that $g_{0}\left(y_{i_{1}}\right)=g_{0}\left(y_{i_{2}}\right)=0$, then $g_{0}$ has $n+1$ distinct zeros $t_{1}, \ldots, t_{n-1}, y_{i_{1}}, y_{i_{2}}$ on $(a, b)$. This is also a contradiction of the hypothesis. 
Therefore there is exactly one double zero $y_{i_{0}}$ of $\tilde{g}_{0}$ such that $g_{0}\left(y_{i_{0}}\right)=0$. Then $g_{0}$ has $n$ distinct zeros $t_{1}, \ldots, t_{n-1}, y_{i_{0}}$ on $(a, b)$. Then for sufficiently small $c>0$ the function $\tilde{g}_{0}-c g_{0}$ has at least $k+2(n-k-2)=n+n-k-4>n-1$ changes of sign on $(a, b)$ because $g_{0}$ does not vanish on exactly $n-k-2$ double zeros of $\tilde{g}_{0}$. Moreover $\tilde{g}_{0}-c g_{0}$ has a further zero in $y_{i_{0}}$ and also a further zero on a neighborhood of $a$, because $g_{0} \cdot \tilde{g}_{0} \geqslant 0$ on $\left[a, t_{k+1}\right]$.

Hence $\tilde{g}_{0}-c g_{0}$ has at least $n+1$ distinct zeros on $[a, b)$. This is a contradiction of the hypothesis of this case. Hence we have shown that $n-k=1$ or $n-k=2$.

We distinguish these two cases:

(i) $n-k=1$. Therefore $\tilde{g}_{0}$ has exactly $n-1$ changes of sign on $(a, b)$. Let $g \in G, g \neq 0$. Then $g(a)=g(b)=0$, because otherwise the function $\tilde{g}_{0}-c g$ has $n$ changes of sign for sufficiently small $c$.

Therefore $g(a)=g(b)$ for all $g \in G$.

Now we proceed as we did in the first case. We choose $n$ distinct points $\left\{v_{l}\right\}_{l=1}^{n}$ satisfying

$$
a<v_{1}<z_{1}<v_{2}<z_{2}<\cdots<v_{n-1}<z_{n-1}<v_{n}<b .
$$

Let $\left\|\tilde{g}_{0}\right\| \leqslant 1$ and $\tilde{g}_{0} \geqslant 0$ on $\left[a, z_{1}\right]$. We choose $\varepsilon>0$ such that

$$
\left\{z_{0}, \ldots, z_{n}\right\} \cap\left[v_{l}-\varepsilon, v_{l}+\varepsilon\right]=\varnothing, \quad l=1, \ldots, n .
$$

We construct an $f \in C[a, b]$ as follows:

(a)

$$
\begin{aligned}
& f(a)=1, \\
& f(x)=(-1)^{l-1} \quad \text { for all } x \in\left[v_{l}-\varepsilon, v_{l}+\varepsilon\right], l=1, \ldots, n \\
& f(b)=(-1)^{n}
\end{aligned}
$$

(b)

$$
\max \left\{-1+\tilde{g}_{0}(x),-1\right\} \leqslant f(x) \leqslant \min \left\{1+\tilde{g}_{0}(x), 1\right\} \text { for all } x \in[a, b] .
$$

Then $\|f-0\|=\left\|f-\tilde{g}_{0}\right\|=1$ and $0, \tilde{g}_{0} \in P(f)$.

It is easy to show that each $g \in P(f)$ has exactly $n-1$ changes of sign. Moreover $g(a)=g(b)=0$ for all $g \in P(f)$. Therefore $a, b \in \operatorname{bd} Z(P(f))$. Applying Lemma 8 to the point $a$ and $\tilde{g}_{0} \in P(f)$ and to the point $b$ and $0 \in P(f)$ we get a contradiction of the hypothesis that there exists a continuous selection.

(ii) $n-k=2$. Therefore, $\tilde{g}_{0}$ has exactly $n-2$ zeros with changes of sign and exactly one double zero $z_{i}$ on $(a, b)$.

We choose $n-1$ distinct points $\left\{v_{l}\right\}_{l=1}^{n-1}$ satisfying

$$
a<z_{1}<v_{1}<z_{2}<v_{2}<\cdots<v_{n-2}<z_{n-1}<v_{n-1}<b .
$$

Let $\left\|\tilde{g}_{0}\right\| \leqslant 1$ and $\tilde{g}_{0} \geqslant 0$ on $\left[a, z_{1}\right]$. We choose $\varepsilon>0$ such that

$$
\left\{z_{0}, \ldots, z_{n}\right\} \cap\left[v_{l}-\varepsilon, v_{l}+\varepsilon\right]=\varnothing, \quad l=1, \ldots, n-1
$$

and $a+\varepsilon<z_{1}$. 
We construct an $f \in C[a, b]$ as follows:

(a)

$$
\begin{aligned}
& f(x)=1 \text { for all } x \in[a, a+\varepsilon], \\
& f(x)=(-1)^{l} \text { for all } x \in\left[v_{l}-\varepsilon, v_{l}+\varepsilon\right], l=1, \ldots, i-1, \\
& f\left(z_{i}\right)=(-1)^{i}, \\
& f(x)=(-1)^{l+1} \text { for all } x \in\left[v_{l}-\varepsilon, v_{l}+\varepsilon\right], l=i, \ldots, n-1, \\
& f(b)=(-1)^{n} ;
\end{aligned}
$$

(b)

$$
\max \left\{-1+\tilde{g}_{0}(x),-1\right\} \leqslant f(x) \leqslant \min \left\{1+\tilde{g}_{0}(x), 1\right\} \text { for all } x \in[a, b] .
$$

Then $\|f-0\|=\left\|f-\tilde{g}_{0}\right\|=1$ and $0, \tilde{g}_{0} \in P(f)$, since $f-0$ has $n+1$ alternating extreme points.

Let $g \in P(f), g \neq 0$. Then it is easy to show that $g$ has at least $n-2$ changes of sign and a double zero at $z_{i}$, since otherwise $g$ has $n$ changes of sign. This would be a contradiction of the hypothesis on $G$.

Since $g(a) \geqslant 0,(-1)^{n} g(b) \geqslant 0$, for sufficiently small $c>0$ the function $\tilde{g}_{0}-c g$ has $n-2$ changes of sign, a double zero at $z_{i}$ and two further zeros on neighborhoods of $a$ and $b$. Since by hypothesis $\left|Z\left(\tilde{g}_{0}-c g\right)\right| \leqslant n$ on $[a, b)$ and on $(a, b]$, the function $\tilde{g}_{0}-c g$ has two zeros at $a$ and $b$. Therefore $g(a)=g(b)=0$ for all $g \in P(f)$ and $a, z_{i}, b \in \operatorname{bd} Z(P(f))$.

Applying Lemma 8 to the point $a$ and $\tilde{g}_{0} \in P(f)$ and to the point $z_{i}$ and $0 \in P(f)$ we get a contradiction of the hypothesis that there exists a continuous selection.

Now we give two examples showing that it is necessary to distinguish the two cases $n-k=1$ and $n-k=2$ in the third part of the above proof.

EXAmple 1. $G:=\left\langle\sin \frac{1}{2} x, \sin x\right\rangle \subset C[0,2 \pi]$. Here $|Z(g)|<2$ for all $g \in G$ on $[0,2 \pi)$ and on $(0,2 \pi]$. The function $\tilde{g}_{0}(x)=\sin x$ has exactly the distinct zeros 0 , $\pi, 2 \pi$ such that $n-k=2-1=1$.

EXAMPLE 2. $G:=\left\langle x^{3},|x|(1-|x|)\right\rangle \subset C[-1,1]$. Here $|Z(g)|<2$ for all $g \in G$ on $[-1,1)$ and on $(-1,1]$. There is no $g \in G$ with three zeros $-1=z_{0}<z_{1}<z_{2}=$ 1 such that $z_{1}$ is a zero with change of sign of $g$ but the function $\tilde{g}_{0}(x)=$ $|x|(1-|x|)$ has exactly the distinct zeros $-1,0,1$ where 0 is a double zero of $\tilde{g}_{0}$. Therefore $n-k=2-0=2$.

Last we give a class of weak Chebyshev subspaces $G$ of $C[a, b]$ satisfying the additional condition that no $g$ in $G, g \neq 0$, has a zero interval. Let $g_{0}$ be a nonnegative function in $C[a, b]$ having no zero interval, but at least two distinct zeros on $[a, b]$. Then for any $g_{0}$ having these properties, the space $G$ spanned by the functions $g_{0}(x), x g_{0}(x), \ldots, x^{n-2} g_{0}(x), x^{n-1} g_{0}(x)$ is a weak Chebyshev space in $C[a, b]$, since each $g$ in $G$ has the representation $g(x)=g_{0}(x) \sum_{i=0}^{n-1} a_{i} x^{i}$ and since the function $\sum_{i=0}^{n-1} a_{i} x^{i}$ has at most $n-1$ changes of sign on $(a, b)$. Since $g_{0}$ has no zero interval, no $g$ in $G, g \neq 0$, has a zero interval. 


\section{REFERENCES}

1. R. C. Jones and L. A. Karlovitz, Equioscillation under nomuniqueness in the approximation of continuous functions, J. Approximation Theory 3 (1970), 138-145. MR \#8899.

2. S. Karlin and W. J. Studden, Tchebycheff systems: With applications in analysis and statistics, Interscience, New York, 1966.

3. A. J. Lazar, P. D. Morris and D. E. Wulbert, Continuous selections for metric projections, J. Functional Analysis 3 (1969), 193-216.

4. G. Nürnberger and M. Sommer, Weak Chebyshev subspaces and continuous selections for the metric projection, Trans. Amer. Math. Soc. 238 (1978), 129-138.

5. Characterization of continuous selections of the metric projection for spline functions, J. Approximation Theory 22 (1978), 320-330.

6. G. Nürnberger, Continuous selections for the metric projection and alternation, J. Approximation Theory (to appear).

7. M. Sommer, Continuous selections of the metric projection for 1-Chebyshev spaces, J. Approximation Theory 26 (1978), 46-53.

8. Characterization of continuous selections for the metric projection for generalized splines, SIAM J. Math. Anal. 11 (1980), 23-40.

9. B. Stockenberg, On the number of zeros of functions in a weak Tchebyshev-space, Math. Z. 156 (1977), 49-57.

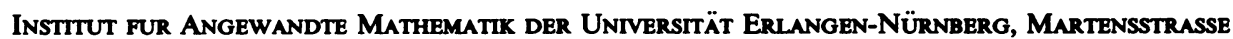
3, 8520 ErLangen, Germany 